\title{
Entanglement Properties and Phase Diagram of the Two-Orbital Atomic Hubbard Model
}

\author{
A. Avella, F. Mancini, G. Scelza \\ Dipartimento di Fisica "E.R. Caianiello" - Unità CNISM di Salerno \\ Università degli Studi di Salerno, 84081 Baronissi (SA), Italy
}

And S. Chaturvedi

School of Physics, University of Hyderabad, Hyderabad 500 134, India

We study the two-orbital Hubbard model in the limit of vanishing kinetic energy. The phase diagram in the $V-J$ plane, with $V$ and $J$ denoting the interorbital hybridization and exchange coupling respectively, at half filling is obtained. A singlet(dimer)-triplet transition is found for a critical value of the ratio $V / J$. The entropy of formation, both in the mode and in the particle pictures, presents a jump at the same critical line in conformity with the suggested relation between criticality and entanglement.

PACS numbers: 71.10.--w, 71.10.Fd, 71.27.+a

\section{Introduction}

The two-orbital Hubbard model has recently come into limelight as a minimal model capable of describing the phenomenon of orbital-selective Mott transition experimentally observed in certain materials [1-3]. This was demonstrated [4] through a simple two-pole approximation within the framework of the composite operator method [5]. In the present work, we report a preliminary study aimed at improving the two-pole approximation by using a new basis, the one that solves the system when reduced to a single atom.

\section{The model}

The Hamiltonian describing the two-orbital Hubbard model in the limit of vanishing kinetic energy (i.e., reduced to a single atom) reads as 


$$
\begin{aligned}
H= & V \sum_{\alpha \neq \beta} c_{\alpha}^{\dagger} c_{\beta}-\mu \sum_{\alpha} c_{\alpha}^{\dagger} c_{\alpha}+U \sum_{\alpha} D_{\alpha} \\
& +U^{\prime} n_{1} n_{2}-\frac{1}{2} J n_{1 \mu} n_{2 \mu}+J \sum_{\alpha \neq \beta} c_{\alpha \uparrow} c_{\alpha \downarrow} c_{\beta \downarrow}^{\dagger} c_{\beta \uparrow}^{\dagger},
\end{aligned}
$$

where $c_{\alpha}^{\dagger}=\left(c_{\alpha \uparrow}^{\dagger}, c_{\alpha \downarrow}^{\dagger}\right)$ is the electronic creation operator in spinorial notation in the orbital $\alpha, D_{\alpha}$ is the double occupancy operator in the orbital $\alpha, n_{\alpha \mu}$ is the $\left(\mu=0\right.$ or $\left.n_{\alpha}\right)$ charge and $(\mu=1,2,3)$ spin density operator in the orbital $\alpha, V$ is the interorbital hybridization, $\mu$ is the chemical potential, $U$ is the intraorbital Coulomb repulsion, $U^{\prime}$ is the interorbital Coulomb repulsion, $J$ is the exchange interorbital interaction. Hereafter, we will use $U$ as the unit of energy and we will fix, as usual, $U^{\prime}=U-2 J$.

\section{Phase diagram and entanglement}

At zero temperature and half filling $(N=2)$, by studying the exact solution in terms of eigenvalues and eigenvectors of $H$, it is possible to show that the system undergoes a phase transition between a singlet (dimer) state $[|\uparrow ; \downarrow\rangle \oplus|\uparrow \downarrow ; 0\rangle]$ and a triplet one $[|\uparrow ; \uparrow\rangle]$ at a critical value of the interorbital hybridization: $V_{\mathrm{c}}=\sqrt{2} J$.

The eigenvalues and eigenvectors in the half-filling sector read as

$$
\begin{aligned}
& |1\rangle=\frac{1}{\sqrt{2}}(|\uparrow \downarrow ; 0\rangle+|0 ; \uparrow \downarrow\rangle), \\
& |2\rangle=\frac{1}{\sqrt{2\left(a^{2}+1\right)}}[a(|\uparrow ; \downarrow\rangle-|\downarrow ; \uparrow\rangle)+|\uparrow \downarrow ; 0\rangle-|0 ; \uparrow \downarrow\rangle], \\
& |3\rangle=\frac{1}{\sqrt{2\left(b^{2}+1\right)}}[b(|\uparrow ; \downarrow\rangle-|\downarrow ; \uparrow\rangle)+|\uparrow \downarrow ; 0\rangle-|0 ; \uparrow \downarrow\rangle], \\
& |4\rangle=|\uparrow ; \uparrow\rangle, \\
& |5\rangle=\frac{1}{\sqrt{2}}(|\uparrow ; \downarrow\rangle+|\downarrow ; \uparrow\rangle), \\
& |6\rangle=|\downarrow ; \downarrow\rangle, \\
& E_{1}=-2 \mu+2 V+U-J, \\
& E_{2}=-2 \mu+2 V+\frac{1}{2}\left(U+U^{\prime}\right)+J-\frac{1}{2} \sqrt{\left(U-U^{\prime}\right)^{2}+16 V^{2}}, \\
& E_{3}=-2 \mu+2 V+\frac{1}{2}\left(U+U^{\prime}\right)+J+\frac{1}{2} \sqrt{\left(U-U^{\prime}\right)^{2}+16 V^{2}}, \\
& E_{4}=-2 \mu+2 V+U^{\prime}-J, \\
& E_{5}=E_{4}, \\
& E_{6}=E_{4},
\end{aligned}
$$

where 


$$
\begin{aligned}
& a=-\frac{1}{4 V}\left(U-U^{\prime}+\sqrt{\left(U-U^{\prime}\right)^{2}+16 V^{2}}\right), \\
& b=-\frac{1}{4 V}\left(U-U^{\prime}-\sqrt{\left(U-U^{\prime}\right)^{2}+16 V^{2}}\right) .
\end{aligned}
$$

In this system, it is also possible to study both the particle entropy [6] and the mode entropy [7]. The particle entropy $S_{\mathrm{p}}$ requires the calculation of the concurrence $C$ :

$$
\begin{aligned}
S_{\mathrm{p}}= & -\frac{1+\sqrt{1-C^{2}}}{2} \log _{2}\left(\frac{1+\sqrt{1-C^{2}}}{2}\right) \\
& -\frac{1-\sqrt{1-C^{2}}}{2} \log _{2}\left(\frac{1-\sqrt{1-C^{2}}}{2}\right), \\
C= & \max \left\{0, \lambda_{1}-\lambda_{2}-\lambda_{3}-\lambda_{4}-\lambda_{5}-\lambda_{6}\right\},
\end{aligned}
$$

where $\left\{\lambda_{i}\right\}$ stands for the square roots of the eigenvalues, taken in descending order of magnitude, of the matrix $\rho D \rho D^{-1} \cdot \rho=\frac{\mathrm{e}^{-\beta H}}{\operatorname{Tr}\left(\mathrm{e}^{-\beta H}\right)}$ is the density matrix of the system ( $\beta$ is the inverse temperature) and $D=-U_{\mathrm{ph}} \mathcal{K}$ is the dualization operator obtained by composing the particle-hole transformation $U_{\mathrm{ph}}$ with the conjugation operator $\mathcal{K}$. The mode entropy $S_{\mathrm{m}}$, on the other hand, requires calculation of the reduced density matrix $\rho_{\beta}$, with respect to a chosen orbital $\alpha(\beta \neq \alpha)$ :

$$
\rho_{\beta}=\sum_{i}\left\langle i_{\alpha}|\rho| i_{\alpha}\right\rangle
$$

where $\left\{\left|i_{\alpha}\right\rangle\right\}$ stands for a complete basis set for the orbital $\alpha$. Then, we simply have $S_{\mathrm{m}}=-\operatorname{Tr}\left(\rho_{\beta} \log \rho_{\beta}\right)$.
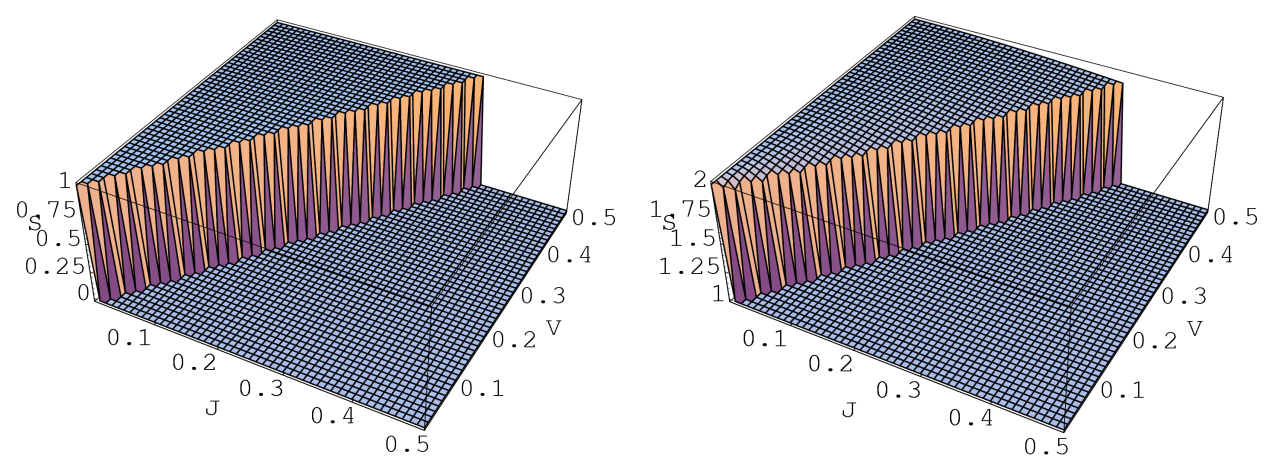

Fig. 1. (left) Particle entropy and (right) mode entropy at $N=2$ and $T=0$ as functions of the interorbital hybridization $V$ and of the exchange interorbital interaction $J$.

In Fig. 1, the particle entropy (left part) and the mode entropy (right part) are reported at $N=2$ and $T=0$ as functions of the interorbital hybridization $V$ and of the exchange interorbital interaction $J$. Both types of entropy show a well defined jump exactly on the line $V=\sqrt{2} J$ where the phase transition occurs. 
However, it is worth noticing that the mode entropy, in contrast to the particle entropy, is not capable of discriminating between a genuine entanglement between substantially different elementary states (a dimer $|\uparrow \downarrow ; 0\rangle$ and a singlet $|\uparrow ; \downarrow\rangle$ ) and the trivial entanglement between states arising from symmetry requirements (the three states of a triplet $|\uparrow ; \uparrow\rangle)$. As a matter of fact, the particle entropy is the only measure correctly accounting for an absolute lack of entanglement in the latter case.

\section{Conclusions}

In conclusion, we have shown that both entanglement measures known in the literature (particle entropy and mode entropy) are capable of capturing the essential physics of the atomic two-orbital Hubbard model. In particular, their jumps can be used for determining the location, in the phase diagram, of the transition line separating the singlet (dimer) state and the triplet.

\section{References}

[1] V.I. Anisimov, I.A. Nekrasov, D.E. Kondakov, T.M. Rice, M. Sigist, Eur. Phys. J. B 25, 191 (2002).

[2] L. de Medici, A. Georges, S. Biermann, Phys. Rev. B 72, 205124 (2005).

[3] M. Ferrero, F. Becca, M. Fabrizio, M. Capone, Phys. Rev. B 72, 205126 (2005).

[4] A. Avella, F. Mancini, S. Odashina, G. Scelza, Physica C 460-462, 1068 (2007).

[5] F. Mancini, A. Avella, Adv. Phys. 53, 537 (2004).

[6] K. Eckert, J. Schliemann, D. Bruß, M. Lewenstein, Ann. Phys. 299, 88 (2002).

[7] P. Zanardi, Phys. Rev. A 65, 042101 (2002). 